\title{
Qing-guo Ma
}

\section{Neural Operation Management: A New Avenue for Productive and Military Operations}

\begin{abstract}
An important effect of technological progress is the increasing replacement of manual labor by mental labor in productive and military operations. The variation of the operator's capabilities in cognition, judgment and decision-making has drawn much attention from operation management researchers. Monitoring and evaluation of these capabilities is especially significant in conditions such as long-time operation, operation with special properties and operation under special circumstances. The military power and economic power are both the key concerns for a nation. The military power depends not only on the weapon system, but also the operators' capabilities of manipulating the system. Similarly, the economic power is not only dependent on advanced machine system, but also the operational capability of the operators. Thus it has become a hot field of research and practice to monitor and assess the operator's physiological and psychological states online based on neural measurement technology, and then to give real time intervention, so as to reduce the occurrence of accidents and increase the operation performance.
\end{abstract}

Keywords: operation management, productive operation, military operation, neural operation management, neuromanagement

\section{Introduction: Operation and operation man- agement}

An operation task consists of three elements, operator, equipment and object of operation. The operators manipulate the equipment and deal with the object to complete an operation. An operator is required to own corresponding skills before carrying out a certain operation task. For some special operations, workers should be trained and assessed in order to be-

Manuscript received May 10, 2014; accepted September 20, 2014

Qing-guo Ma $(\bowtie)$

Department of Management Science and Engineering, Zhejiang University, Hangzhou 310027, China

Email: maqingguo3669@zju.edu.cn come qualified operators. Nowadays, operations are mainly categorized by the features of the equipment and the goals of operations. The operations with different goals constitute an operation chain and then operation chain network and finally contribute to the achievement of overall goals of the productive or military tasks (see Figure 1).

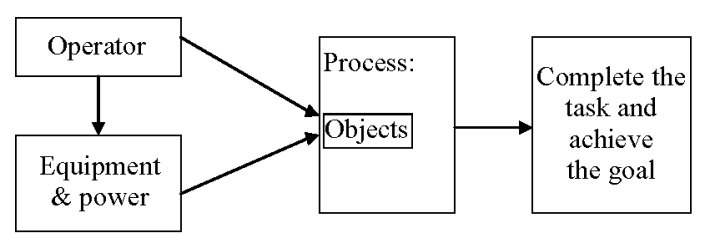

Figure 1. The flowchart of an operation.

Engineering operation management is the sub-field of engineering management. In broad sense, productive (engineering) operation includes enterprise operation (senior), production planning operation (middle) and equipment manipulation (junior) while in narrow sense it mainly focus on the management of the field working operation, such as the manipulation by the production line, or manipulating the weapon system.

The field working operation comprises a variety of operations with different technical features and functions. The goals of an enterprise are achieved by the successful deployment of a series of operation tasks. Similarly, the win of a battle also depends on the success of a series of operation tasks involved.

\section{Key of operation management: the opera- tor's cognition}

The strengths of productivity and military power are both the key concerns for any nation. There exists a misunderstanding that weapons determine the military power. Though weapons are important, they are not the only determinants. Be- 
sides the weapons, the human who manipulate the weapons and equipment are equally important. Only when advanced weapons and skilled operators are highly coordinated can we produce a powerful military force. Likewise, the productivity of a country depends on both equipment and the operators.

The two factors, equipment and operator, can be further divided into four key sub factors. (1)The responsibility, attitude and commitment of the operators; (2)The skills and proficiency of the operators; (3)The techniques, tactics and methods of manipulating the equipment; (4)The advancement and reliability of equipment.

The first three factors are managerial issues while the last is an equipment issue. These four factors are the focus of scholarly attention in the operation management whether in the management of productive operation, engineering operation, service operation or military operation. The highlight of operation management has always being the coordination of operator's action and the equipment, and the scientific handling of operation objects in order to complete an operation safely and efficiently.

Due to technological progress, the manual labor is increasingly replaced by mental labor in the productive and military operations. The operation process and performance are more susceptible to the operators' physiological, psychological state and other social factors. In the process of operation, an operator need to judge on how to operate based on the goal and environment of operation, and then perform the operation strategies to achieve the final goals. Hence, ensuring an appropriate level of cognitive ability is the key to successful operation. Therefore, the variations of the operators' emotion and capabilities of cognition and judgment have become the focus of scholarly attention recently. If we overlook this point and insist on traditional management system to cope with operations featured by mental work, we'll fall behind in this new trend of research of practice.

Three elements are considered to have significant influence on human cognition. First, the operators themselves, such as their physiological state (e.g., level of attention, fatigue and health condition), emotional state (e.g., level of pleasure, excitement and tension) and familiarity of the operation; second, the cognitive objects, such as human-computer interaction interface, among which prominent clues might draw more attention; third, the environment of cognition, including geographical environment, space, temperature, humidity, illumination, noise, etc.

In traditional operation management, the equipment's reliability has drawn much attention, while people has been treated as an omnipotent one who is energetic, focused and do not easily make mistakes during work. However, as a real people, an operator is restrained by a variety of physiological, psychological and social factors. For example, in a highly risky military operation, a soldier is more likely to be faced with the problems of cognitive decline, inattention, short-term memory damage and weakened judgment ability, which greatly affect the operation performance. Therefore, it is of vital importance to monitor and assess the operators' physiological and psychological states in special tasks and environments, for example, high temperature, high noise and hazardous environment.

Since human factor is such a crucial issue, how can we study the reliability of human beings? Traditional operation management methods and tools are no longer feasible to look into this problem. However, neuroscience and other life sciences provide a new perspective for human reliability analysis in operation, which offers a great avenue for operation management researches.

\section{Neural operation management}

The development of platform for the science and technology research often plays a revolutionary role in promoting the development of science and technology. In the past, it was difficult to obtain the mental load data of the operators due to the lack of non-intrusive physiological measurement technology and instrument. Therefore it was difficult to study the operators' mental workload, cognitive and emotional state, judgment and decision-making ability. But now the advance of technologies on measuring human brain activities and other physiological activities, such as electroencephalogram (EEG), event-related potentials (ERP) and eye tracking technology, has enabled us to delve into the aforementioned issues (Parasuraman \& Rizzo, 2008; Parasuraman \& Wilson, 2008).

A large body of literature has been focused on human cognitive and mental load since then (Dussault, Jouanin, Philippe \& Guezennec, 2005; Smith, Gevins, Brown, Karnik $\& \mathrm{Du}, 2001)$. A number of other issues have also been investigated, for example, the variation of brain network during a day, the training of operators based on brain monitoring (Ayaz, Shewokis, Bunce, 2012), and the influence of emotion on operation tasks (Ma, Sun, Fu, Zhao \& Guo, 2013). Moreover, Ma et al. explored the method of work procedure improvement based on mental and physical workload (Ma et al., 2013), and the neural responding mechanism towards safety signs in operation environment (Ma, Jin, \& Wang, 2010).

The United States of America has always been laying emphasis on the application of physiological measurement technology on military operation. Georgia Institute of Technology developed an innovative technology known as the Georgia Tech Wearable Motherboard (GTWM), which provided a systematic way of monitoring the vital signs of humans during operation (Gopalsamy, Park, Rajamanickam, \& Jayaraman, 1999). The Medical Research and Material Command developed a complex wearable computerized system, the "Warfighter Physiological Status Monitoring" with an array of biosensors embedded in the soldier's uniform integrated with a database management system and a decision support system that will provide assistance (Hoyt, Reifman, Coster, \& Buller, 2002). The Defense Advance Research Projects Agency (DARPA) "Augmented Cognition" program has been developing innovative technologies that 
aim at transforming human-computer interactions by making information systems adapt to the changing capabilities and limitations of the user (John St., Kobus, Morrison, \& Schmorrow, 2004). Moreover, Quantum Applied Science and Research, Inc. developed a dry-electrode EEG monitoring system used for analyzing the mental states of soldiers on battlefield and providing assistance for command (see Figure 2).

So far, the advance of brain cognitive science and brain-computer interface (BCI) technology has contributed to emergence of a number of interdisciplines, to name but a few, neuroeconomics (Camerer, Loewenstein, \& Prelec, 2005), neuromanagement (Ma \& Wang, 2006), neuro-industrial engineering (Ma, Fu, \& Bian, 2012; Ma \& Wang, 2006), neuroengineering (Hoag, 2003), and neuro-engineering management (Ma, 2012). It is viable to investigate the operator's physical and mental states, such as cognitive capacity, mental workload and emotional changes, and then improve the operation process based upon that. This new trend in operation management might be called neural operation management, which is a branch of neuromanagement. The findings in this new research field will be helpful to reduce accidents and enhance operation performance.

\section{The growing points of neural operation management}

The growth of neural operation management centers on the rationality of human cognitive ability and mental workload during operation. A key step after collecting the physiological signals is to filter noise signals and construct mathematical models that are able to transform the original signals into indexes that reflect the cognitive state, emotional state, decision-making ability or mental fatigue state. Based upon human physiological information as well as the information of the operating equipment, the managers are able to make

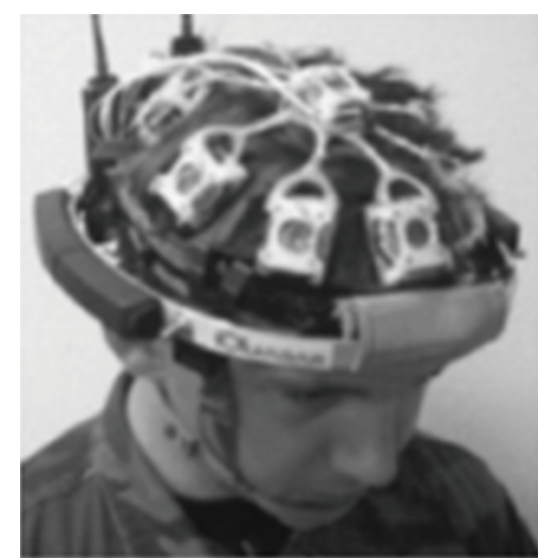

(a) The sensors inside the helmet apposite adjustments, for example, change the operator, offer the operator a short rest, alter operation methods, or improve the equipment, and thereby improve efficiency, reduce accidents and optimize the whole work process (Ma, Shang, $\mathrm{Fu}$, \& Chen, 2012; Ma, Sun, Fu, Zhao, \& Chen, 2013).

There's a variety of topics in this burgeoning field that merit close attention, which could be broadly divided into four categories.

4.1 Operators' physiological and psychological state under different operation contents

It accentuates the impact of the duration, category, and content of an operation task on human physiological, psychological and cognitive states. The issues include mental fatigue in long-time and monotonous work, the attention variation of operators in special workstations (e.g., tower crane driver, power system dispatcher and airport control tower operator); physical and mental workload modeling under diverse work contents; psychological pressure assessment in hazardous article operation.

4.2 Operators' physiological and psychological state under different operation environments

This field of research highlights the potential impact of operation environment, which is featured by geography, space, temperature, moisture, illumination, noise, etc., on operators. The issues include neural processing mechanism towards voice and optical signals; cognitive ability evaluation in high noise, high temperature, underground or low oxygen environment; psychological pressure and decision-making ability under emergency (e.g., flood and fire hazard).

4.3 Operation method improvement based on operators' physiological and psychological state

Given the information of the operator's physiological and

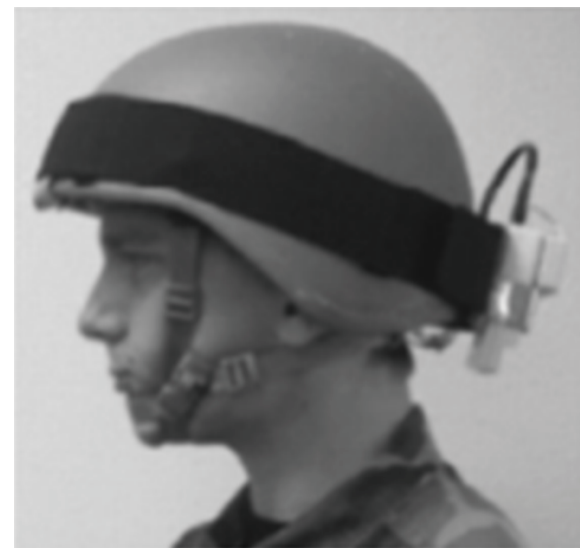

(b) The helmet and the signal emission box

Figure 2. The dry-electrode EEG helmet. 
psychological state, it's viable to give certain intervention and improve the operation process, for example, provide early warning of mental fatigue for operators, deploy recovery training on physical and mental fatigue, and improve the standard operation procedure and work system.

4.4 Human-machine interaction based on brain cognitive science

To achieve efficient production and military operation performance, the operator and machine has to be highly coordinated. Therefore the research on human-machine interaction is essential in neural operation management. Information system interface design, high-pressure vessel control console interface design and vital instrument interface design are all important issues in this field of research and practice.

\section{Conclusions}

Neural operation management is a deliberate merger of neuroscience and operation management with the goal of improving operation method, reducing the occurrence of accidents and increasing operation performance. A second major goal is to advance understanding of neurophysiological features underlying operation and operation management. With great emphasis on operators' physiological and psychological states, neural operation management offers a new avenue for productive and military operations in the aim of escalating economic and military power.

\section{References}

Ayaz, H., Shewokis, P. A., Bunce, S., Izzetoglu, k., Willems, B., \& Onaral, B. (2012). Optical brain monitoring for operator training and mental workload assessment. Neuroimage, 59(1), 36-47.

Camerer, C., Loewenstein, G., \& Prelec, D. (2005). Neuroeconomics: How neuroscience can inform economics. Journal of Economic Literature, 9-64.

Dussault, C., Jouanin, J. C., Philippe, M., \& Cuezennec, C.Y. (2005). EEG and ECG changes during simulator operation reflect mental workload and vigilance. Aviation, Space, and Environmental Medicine, 76(4), 344-351.
Gopalsamy, C., Park, S., Rajamanickam, R., \& Jayaraman, S. (1999). The Wearable Motherboard ${ }^{\mathrm{TM}}$ : The first generation of adaptive and responsive textile structures (ARTS) for medical applications. Virtual Reality, 4(3), 152-168.

Hoyt, R. W., Reifman, J., Coster, T. S., \& Buller, M.J. (2002). Combat medical informatics: Present and future. In Proceedings of the AMIA Symposium. American Medical Informatics Association, 335.

Ma, Q. G. (2012). The framework of engineering management and neuro-engineering management. Science \&Technology Progress and Policy, 29(18), 9-12.

Ma, Q., \& Wang, X. (2006). Cognitive neuroscience, neuroeconomics and neuromanagement. Management World, 10, 139-149.

Ma, Q., Fu, H., \& Bian, J. (2012). Neuro-industrial engineering: the new stage of industrial engineering. Management World, 6, 163$168,179$.

Ma, Q., Shang, Q., Fu, H., Chen, F. (2012). Mental workload analysis during the production process: EEG and GSR activity. Applied Mechanics and Materials, 220, 193-197.

Ma, Q., Sun, X., Fu, H., Zhao, D., \& Guo, J. (2013). Manufacturing process design based on mental and physical workload analysis. Applied Mechanics and Materials, 345, 482-485.

Ma, Q., Jin, J., \& Wang, L. (2010). The neural process of hazard perception and evaluation for warning signal words: Evidence from event-related potentials. Neuroscience Letters, 483(3), 206-210.

Parasuraman, R., \& Rizzo, M. (2008). Neuroergonomics: The Brain at Work. Oxford University Press, Inc.

Parasuraman, R., \& Wilson, G. F. (2008). Putting the brain to work: Neuroergonomics past, present, and future. Human Factors: The Journal of the Human Factors and Ergonomics Society, 50(3), 468-474.

Park, B., Kim, J. I., Lee, D., Jeong, S.O., \& Park, H.J. (2012). Are brain networks stable during a 24-hour period. Neuroimage, 59(1), 456466.

Smith, M. E., Gevins, A., Brown, H., Kamik, A., \& Du, R. (2001). Monitoring task loading with multivariate EEG measures during complex forms of human-computer interaction. Human Factors: The Journal of the Human Factors and Ergonomics Society, 43(3), 366-380.

John St., M., Kobus, D. A., Morrison, J. G., \& Schmorrow, D. (2004). Overview of the DARPA augmented cognition technical integration experiment. International Journal of Human-Computer Interaction, 17(2), 131-149. 